\title{
Neurofascin 140 Is an Embryonic Neuronal Neurofascin Isoform That Promotes the Assembly of the Node of Ranvier
}

\author{
Ao Zhang, ${ }^{1}$ Anne Desmazieres, ${ }^{1}$ Barbara Zonta, ${ }^{1}$ Shona Melrose, ${ }^{1}$ Graham Campbell, ${ }^{1}$ Don Mahad, ${ }^{1}$ Qiushi Li, ${ }^{1}$ \\ Diane L. Sherman, ${ }^{1}$ Richard Reynolds, ${ }^{2 \star}$ and Peter J. Brophy ${ }^{1 *}$ \\ ${ }^{1}$ Centre for Neuroregeneration, The Medical School, University of Edinburgh, Edinburgh EH16 4SB, United Kingdom, and ${ }^{2}$ Centre for Neuroinflammation \\ and Neurodegeneration, Faculty of Medicine, Imperial College London, London W12 0NN, United Kingdom
}

\begin{abstract}
Rapid nerve conduction in myelinated nerves requires the clustering of voltage-gated sodium channels at nodes of Ranvier. The Neurofascin ( $N f a s c$ ) gene has a unique role in node formation because it encodes glial and neuronal isoforms of neurofascin (Nfasc155 and Nfasc186, respectively) with key functions in assembling the nodal macromolecular complex. A third neurofascin, Nfasc140, has also been described; however, neither the cellular origin nor function of this isoform was known. Here we show that Nfasc140 is a neuronal protein strongly expressed during mouse embryonic development. Expression of Nfasc140 persists but declines during the initial stages of node formation, in contrast to Nfasc155 and Nfasc186, which increase. Nevertheless, Nfasc140, like Nfasc186, can cluster voltage-gated sodium channels (Nav) at the developing node of Ranvier and can restore electrophysiological function independently of Nfasc155 and Nfasc186. This suggests that Nfasc140 complements the function of Nfasc155 and Nfasc186 in initial stages of the assembly and stabilization of the nodal complex. Further, Nfasc140 is reexpressed in demyelinated white matter lesions of postmortem brain tissue from human subjects with multiple sclerosis. This expands the critical role of the $\mathrm{Nfasc}$ gene in the function of myelinated axons and reveals further redundancy in the mechanisms required for the formation of this crucial structure in the vertebrate nervous system.
\end{abstract}

Key words: axon initial segment; myelin; neurofascin; Nfasc140; node of Ranvier

\section{Introduction}

Myelinated nerves can conduct nerve impulses rapidly because voltage-gated sodium channels are concentrated at the nodes of Ranvier between successive myelinated segments. Nodes are the sites of action potential propagation due to the assembly of a macromolecular complex, including voltage-gated sodium channels. Components of the nodal complex include $\beta \mathrm{IV}$-spectrin, ankyrinG, contactin (in the CNS), NrCAM and gliomedin (in the PNS), and a neuronal isoform of neurofascin, Nfasc186 (Davis et al., 1996; Berghs et al., 2000; Tait et al., 2000; Jenkins and Bennett, 2002; Yang et al., 2004; Eshed et al., 2005; Bekku and Oohashi, 2010). Flanking the nodes in both the CNS and PNS are the paranodal axoglial junctions where the terminal loops of the my-

Received Aug. 25, 2014; revised Dec. 1, 2014; accepted Dec. 8, 2014.

Author contributions: A.Z., A.D., B.Z., and P.J.B. designed research; A.Z., S.M., G.C., Q.L., and D.L.S. performed research; A.Z., D.M., R.R., and P.J.B. analyzed data; R.R. and P.J.B. wrote the paper.

This work was supported by the Wellcome Trust and the Multiple Sclerosis Society 910/09 to R.R. The human postmortem MS tissues and associated clinical and neuropathological data were supplied by the Multiple Sclerosis Society Tissue Bank (funded by the Multiple Sclerosis Society, registered charity 207495). We thank Stuart Fleming and Heather Anderson for excellent technical support; and Dr. U. Suter (ETH, Zurich) for providing the PLPCreERT2 line.

The authors declare no competing financial interests.

${ }^{*}$ R.R. and P.J.B. contributed equally to this study as joint senior authors.

This article is freely available online through the J Neurosci Author Open Choice option.

Correspondence should be addressed to Dr. Peter J. Brophy, Centre for Neuroregeneration, The Medical School, University of Edinburgh, 49 Little France Crescent, Edinburgh EH16 4SB, UK. E-mail: Peter.Brophy@ed.ac.uk.

DOI:10.1523/JNEUROSCI.3552-14.2015

Copyright $\odot 2015$ Zhang et al.

This is an Open Access article distributed under the terms of the Creative Commons Attribution License (http://creativecommons.org/licenses/by/3.0), which permits unrestricted use, distribution and reproduction in any medium provided that the original work is properly attributed. elin sheath physically interact with the axon (Sherman and Brophy, 2005; Salzer et al., 2008). Axon-glial interaction at the paranode is mediated by an adhesion complex between the glial isoform of neurofascin, Nfasc155, and the axonal proteins caspr (also known as paranodin) and contactin (Einheber et al., 1997; Menegoz et al., 1997; Tait et al., 2000; Bhat et al., 2001; Boyle et al., 2001; Kazarinova-Noyes et al., 2001; Charles et al., 2002; Sherman et al., 2005).

The Neurofascin (Nfasc) gene is essential for the assembly of the nodal complex (Sherman et al., 2005). Nfasc has a unique role by encoding a glial isoform, neurofascin 155 (Nfasc155) and a neuronal isoform, neurofascin 186 (Nfasc186) (Davis and Bennett, 1993, 1994; Davis et al., 1993, 1996; Collinson et al., 1998; Tait et al., 2000), both of which have key roles in clustering sodium channels (Sherman et al., 2005; Zonta et al., 2008; Feinberg et al., 2010; Thaxton et al., 2011). A third isoform was also described in the original discovery of these proteins by Bennett and colleagues (Davis et al., 1993). This protein has an apparent size by SDS-PAGE of $140 \mathrm{kDa}$ and was consequently named neurofascin 140 (Nfasc140). Nevertheless, little progress has been made in determining the function of Nfasc140.

Given the complementary roles of glial Nfasc155 and neuronal Nfasc186 in the assembly of nodal complexes, we wished to determine whether Nfasc140 might have a related function. First, we determined that Nfasc140 is a neuronal protein. We then investigated whether Nfasc 140 was reexpressed in demyelinated axons in multiple sclerosis (MS) lesions. To address its function, we used a transgenic rescue approach, as described previously 
(Sherman et al., 2005). Nfasc140 is as capable as neuronal Nfasc186 of clustering sodium channels and other members of the nodal complex at nodes of Ranvier in both the PNS and the CNS. Hence, we have identified a third member of the neurofascin family with a complementary role to Nfasc155 and Nfasc186 in the assembly of the node of Ranvier. The fact that transgenic Nfasc140 is also targeted to the axon initial segment (AIS) and rescues the normal localization of $\mathrm{NrCAM}$ also supports the view that Nfasc140 and Nfasc186 have related functions in stabilizing this domain (Zonta et al., 2011).

\section{Materials and Methods}

Isolation of Nfasc140 cDNA and detection of Nfasc186, Nfasc155, and Nfasc140 isoforms by RT-PCR. RNA was extracted from mouse hind brain at E15 and subjected to RT-PCR as described previously (Tait et al., 2000) using 5'-ATGGCCAGGCAGCAGGCGCCAC-3' and 5'-CCAT CTATTCCCTTGCCTGA- $3^{\prime}$ forward and reverse primers, respectively, which span the full coding regions of all neurofascins. The gel-purified product of $3.2 \mathrm{~kb}$ was sequenced. To detect Nfasc186, Nfasc155, and Nfasc140 mRNAs in P21 hind brain or optic nerve, RNA primers spanning the second and fourth FNIII repeats common to all three isoforms were used (5'-CGCTACATTGTCAAGTGGCG-3' and 5'AAGCGGT AACGGGACACGGG-3' forward and reverse primers, respectively). The predicted sizes for Nfasc 186 or Nfasc140 mRNA were $411 \mathrm{bp}$ and $732 \mathrm{bp}$ for Nfasc155.

Animals. All animal work conformed to UK legislation (Scientific Procedures) Act 1986, and to the University of Edinburgh Ethical Review Committee policy. Thy1Nfasc186 transgenic mice expressing full-length Nfasc186 with a FLAG tag at its C terminus under the control of the Thy1.2 promoter have been described and are here abbreviated as T186 (Zonta et al., 2011). The Nfasc140 cDNA with a FLAG tag at its C terminus was cloned into the XhoI site of the pTSC21k vector (Lüthi et al., 1997), released by digestion with NotI and transgenic mice expressing Nfasc140 under the control of the Thy1.2 promoter (T140) were similarly generated by pronuclear injection. The tamoxifen-inducible Thy1CreERT2 and PLPCreERT2 lines and the floxed Neurofascin line $\left(N f a s c^{f l / f l}\right)$ have been described (Leone et al., 2003; Zonta et al., 2011). Induction of recombination using tamoxifen has been described (Zonta et al., 2011). All lines were back-crossed to at least F6 to a C57BL/6 background. To express transgenic Nfasc140 and Nfasc186 on a neurofascin-null background, the $\mathrm{T1} 140$ and $\mathrm{T} 186$ lines were interbred with $\mathrm{Nfasc}^{+/-}$mice to generate T140/Nfasc ${ }^{-1-}$ and T186/Nfasc ${ }^{-1-}$ mice.

Antibodies, microscopy, Western blots, yeast two-hybrid, electrophysiology, and behavioral analysis. Immunostaining of teased quadriceps, spinal cord ventral funiculus nerves, and cerebellar sections was as described previously (Sherman et al., 2005; Zonta et al., 2011). Most antibodies used to stain these preparations have been described (Zonta et al., 2008, 2011) with the addition of anti-GFAP (GA-5, dilution 1:100, Sigma) and anti-IBA1 (dilution 1:1000, Wako). Samples were mounted in Vectashield (Vector Laboratories). For confocal microscopy, we used a Leica TCL-SL confocal microscope and $63 \times$ objective, numerical aperture 1.4, and Leica proprietary software. Conventional fluorescence microscopy was performed using an Olympus BX60 microscope and 40X objective lens, numerical aperture 0.75 , and images were captured using a Hamamatsu Orca-ER camera and Improvision Openlab software. All figures were prepared using Adobe Photoshop version 7.0 and were not subjected to any subsequent image processing. Western blotting was performed as described previously (Sherman et al., 2005). Yeast twohybrid interactions were tested exactly as previously described (Sherman et al., 2001; Gunn-Moore et al., 2006). Electrophysiological measurements of nerve conduction velocity were performed on acutely isolated quadriceps nerves and performance on rotarod as described previously (Court et al., 2004; Sherman et al., 2012). All quantitative measurements were from an equal number of males and females.

MS postmortem tissues. All postmortem tissues were obtained from the UK Multiple Sclerosis Society Tissue Bank at Imperial College. The tissues were all collected via a prospective donor scheme following fully informed consent (08/MRE09/31). The demographic data and clinical
Table 1. Details of MS and control subjects used in this study ${ }^{a}$

\begin{tabular}{lllllll}
\hline Case no. & Sex & Age (yr) & PMD (h) & Disease duration (yr) & Cause of death & Course \\
\hline C032 & M & 88 & 22 & - & Prostate cancer & - \\
C036 & M & 68 & 30 & - & Fibrosing alveolitis & - \\
C037 & M & 84 & 5 & - & Bladder cancer & - \\
C045 & M & 77 & 22 & - & Pneumonia & - \\
MS200 & F & 43 & 20 & 19 & Urinary tract infection & SP \\
MS203 & F & 53 & 17 & 27 & Multiple sclerosis & SP \\
MS207 & F & 46 & 10 & 25 & Pneumonia & SP \\
MS230 & F & 42 & 31 & 19 & Multiple sclerosis & SP \\
MS256 & F & 53 & 21 & 24 & Pneumonia & SP \\
MS527 & M & 47 & 10 & 25 & Pneumonia & SP \\
\hline
\end{tabular}

${ }^{a}$ PMD, Postmortem delay; SP, secondary progressive MS.

and neuropathological features of the secondary progressive MS cases and controls are shown in Table 1. The clinical diagnosis of MS was confirmed based on the patient history and a detailed neuropathological analysis, as described previously (Reynolds et al., 2011). MS white matter lesions were classified according to the UK MS Society Tissue Bank's published criteria (Reynolds et al., 2011) and tissue blocks chosen containing chronic active lesions. The presence of chronic active lesions was confirmed in serial sections as described previously (Mahad et al., 2009). Briefly, snap frozen tissue blocks and cryostat sections, cut to a thickness of $15 \mu \mathrm{m}$, were kept at $-80^{\circ} \mathrm{C}$ for storage. Sections were air dried for an hour before being fixed in a cold solution of $4 \%$ PFA. Antigen retrieval using 10 mM EDTA, $\mathrm{pH}$ 8, was performed, and a blocking solution of $1 \%$ normal goat serum was applied. Primary antibodies HLA-DP, DQ, DR (dilution 1:200, Dako), PLP (dilution 1:500, AbD Serotec), and total neurofilament (dilution 1:1000, Thermo Scientific) were applied at room temperature for $90 \mathrm{~min}$. Biotinylated secondary antibodies were added for $30 \mathrm{~min}$ and visualized using DAB. Sections were immersed in a hematoxylin solution for $1 \mathrm{~min}$ followed by mounting. The borders of the lesions were scored on the snap frozen tissue block with a scalpel blade, which allowed separation of the lesion and normal-appearing white matter when cryosectioning at $50 \mu \mathrm{m}$, and $50-150 \mathrm{mg}$ tissue isolated for subsequent Western blotting.

\section{Results}

Nfasc140 is an embryonic neurofascin isoform in neurons

Western blotting of lysates from mouse hindbrains at different ages showed that a neurofascin band at $140 \mathrm{kDa}$ was expressed in the embryonic mouse hind brain, whereas Nfasc186, although also present, became more abundant at the onset of myelination when Nfasc140 was downregulated (Fig. 1A). The identity of the band that has an apparent size larger than Nfasc155 between Nfasc140 and Nfasc186 at E15 is unknown. Nfasc155 expression is associated with the initiation of myelination (Collinson et al., 1998), and this was reflected in its increased abundance at P8 (Fig. $1 A)$. Because the band with a mobility of $140 \mathrm{kDa}$ was very abundant at E15, we purified hindbrain RNA at E15 and performed RT-PCR to isolate full-length cDNA clones of Nfasc140. These clones were sequenced, and the deduced protein domain structure was compared with the two well-characterized neurofascin isoforms (Fig. 1B). This confirmed that Nfasc140 lacked the mucin and FNIII5 domains characteristic of neuronal Nfasc186, and the FNIII3 domain unique to Nfasc155 as found previously by Bennett and coworkers (Davis et al., 1993) (Fig. 1B). Western blot analysis confirmed that Nfasc140 lacked the mucin and FNIII5 domains and the FNIII3 domain unique to Nfasc155 (Fig. 1C). The lower proteins recognized by the extracellular domainspecific antibodies, NFF3 and MNF2, but not by NFC (which recognizes the identical intracellular $\mathrm{C}$ terminus present in neurofascins 140,155 , and 186), is likely to represent forms of these proteins that have been subject to extracellular cleavage (Maier et al., 2006). To determine whether Nfasc140 was expressed by neu- 
A

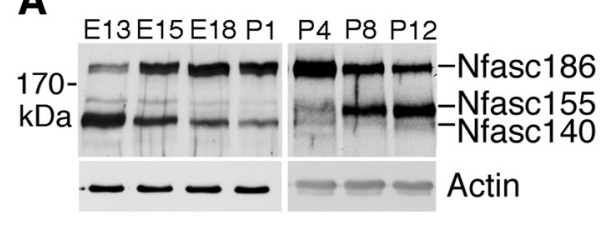

B

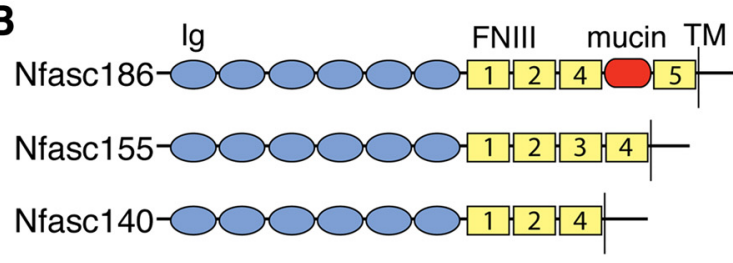

C

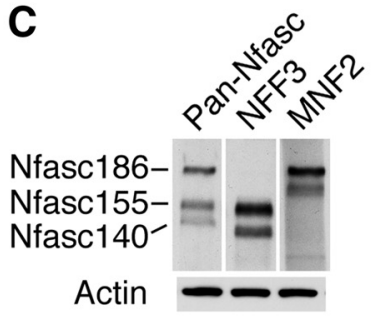

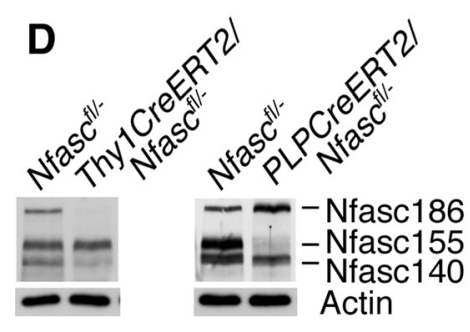

Figure 1. Nfasc140 is a neuronal isoform of neurofascin. $A$, Developmental Western blot of hind brain lysates on two separate gels using a pan anti-neurofascin antibody (NFC) that recognizes the C terminus of the cytoplasmic domain shows abundant but declining expression of Nfasc140 relative to Nfasc186 in the embryo up until birth (E13-P1), which declines further postnatally at the onset of myelination (P4-P12) when glial Nfasc155 becomes prominent. Actin was used to ensure equal loading in each group. $\boldsymbol{B}$, The domain structure of the three neurofascin isoforms. Sequencing of the Nfasc140 RT-PCR product generated from E15 RNA showed that it lacked the mucin and FNIIII domains characteristic of neuronal Nfasc186 or the FNIIII3 domain unique to Nfasc155. Ig, Immunoglobulin-like domain; FNIII, fibronectin Type III domain; TM, transmembrane domain. C, Western blots of hind brain lysates at P45 confirmed that Nfasc140 lacked the FNIII3 and mucin/FNIII5 domains found in Nfasc155 and Nfasc186, respectively. As described previously (Tait et al., 2000), the pan anti-neurofascin antibody (NFC) was raised against a region at the extreme $\mathrm{COOH}$ terminus of the intracellular domain common to Nfasc186, Nfasc155, and Nfasc140 (CGNESSEATSPVNAAIYSLA), the second (NFF3) to the third FNIII domain specific to Nfasc155 (CLWVSQKRQQASFPGDRPR), and the third (MNF2) to a sequence within the mucin domain specific to Nfasc186 (CTESPTTTTGTKIHETAPDEQS). Note the presence of Nfasc 155 and Nfasc 186 bands that lack the C terminus of the full-length proteins. Actin was the loading control. D, Neuron- and glia-specific Cre-mediated inactivation of the Nfasc gene shows that Nfasc 140 is a neuronal protein. Mice heterozygous for a floxed allele of Nfasc and heterozygous null for Nfasc and either expressing the transgene encoding a tamoxifen-inducible neuronal Cre (Thy $1\left(\mathrm{CreERT2/Nfas} \mathrm{fl}^{f /-}\right.$ ) or glial Cre (PLPCreERT2/Nfasc ${ }^{f l /-}$ ) were treated with tamoxifen at P30, and hind brain lysates were analyzed by Western blot 2.5 months later using the pan anti-neurofascin antibody (NFC). Controls $\left(\right.$ Nfas $\left.^{f l /-}\right)$ lacked Cre. Actin was the loading control. E, Neurofascins are not expressed in astrocytes or microglia. Double-label immunofluorescence using the pan anti-neurofascin antibody (NFC) (green) with either anti-GFAP for astrocytes (red) or anti-IBA1 for microglia (red) reveals neurofascins at axonal nodes and paranodes (arrows) but not in either astrocytes or microglia. Nfasc 186 is not expressed in olgodendrocytes. Double-label immunofluorescence using the Nfasc186-specific antibody (MNF2) (green) and the oligodendrocyte-specific antibody APC shows labeling of axonal nodes (arrow) but no Nfasc186 in oligodendrocytes (red). Scale bar, $5 \mu \mathrm{m}$. $\boldsymbol{F}$, Neither Nfasc186 nor Nfasc140 is expressed in non-neuronal cells. RT-PCR was used to discriminate between mRNAs encoding either Nfasc186 and Nfasc140 (411 bp) or Nfasc155 (732 bp) in total hind brain (HB) RNA or in optic nerve (ON) RNA.

rons or myelin-forming glia, we conditionally inactivated the $\mathrm{Nfasc}$ gene in either neurons or oligodendrocytes by tamoxifen-inducible Cre-mediated recombination using mice carrying a floxed allele of Nfasc and either neuronal-specific (Thy1CreERT2) or glialspecific (PLPCreERT2) Cre lines. Loss of Nfasc186 using the neuron-specific Cre together with the considerable depletion of Nfasc140 suggested that the latter was primarily neuronal in origin (Fig. 1D). This view was supported by the fact that only Nfasc155 was completely depleted by the glial-specific Cre (Fig. 1D). The persistence of some Nfasc140 after neuron-specific ablation may reflect the fact that Nfasc140 has a longer half-life than Nfasc186 (Zonta et al., 2011).

Further evidence that Nfasc140 is a neuronal not a glial protein is shown in Figure $1 \mathrm{E}, \mathrm{F}$. Sections from mouse cerebellum were double-labeled with the pan anti-neurofascin antibody (Pan-Nfasc, NFC) that recognizes Nfasc186, Nfasc155, and Nfasc140 and either anti-GFAP (for astrocytes) or IBA1 (for microglia). This shows staining of axonal nodes and paranodes as expected but no neurofascin staining in either astrocytes or microglia. (Fig. $1 E$ ). To confirm that Nfasc 186 was not expressed by oligodendrocytes, we double stained with the Nfasc186-specific MNF2 antibody and anti-APC (for oligodendrocytes) showing Nfasc186 staining in axonal nodes as expected (Tait et al., 2000), but no staining in oligodendrocytes. Hence, Nfasc186 is not expressed in oligodendrocytes (Fig. 1E).
Finally, to exclude the possibility that Nfasc140 is expressed in neural cells other than neurons, we used primers that span the second and fourth FNIII repeats that are present in Nfasc186, Nfasc155, and Nfasc140 in RT-PCR of RNA purified from wholemouse hind brain or optic nerve (Fig. $1 B$ ). Postnatal optic nerve was chosen because it contains a variety of neural cells, including oligodendrocytes, astrocytes, and microglia, but no neuronal cell bodies, and therefore no neuronal mRNA. The primers were chosen to discriminate between isoforms where the second and fourth FNIII repeats are contiguous (Nfasc186 and Nfasc140) from mRNA where these two domains are separated by FNIII3 (Nfasc155) (Fig. 1B). The predicted size of the products derived from Nfasc 186 and Nfasc140 mRNA is identical (411 bp) and smaller than that from Nfasc155 (732 bp). Both are readily detectable in hind brain. However, only the product derived from Nfasc 155 was obtained from optic nerve (Fig. 1F).

Hence, Nfasc140 is a neuronal neurofascin isoform that is expressed somewhat earlier than its neuronal counterpart, Nfasc186. However, although it persists, Nfasc140 is downregulated as myelination proceeds, in contrast to Nfasc186. This suggests that Nfasc140 has a role in the earliest stages of sodium channel clustering, whereas Nfasc186 has the primary role in neurons during the period of most active myelination. 
A
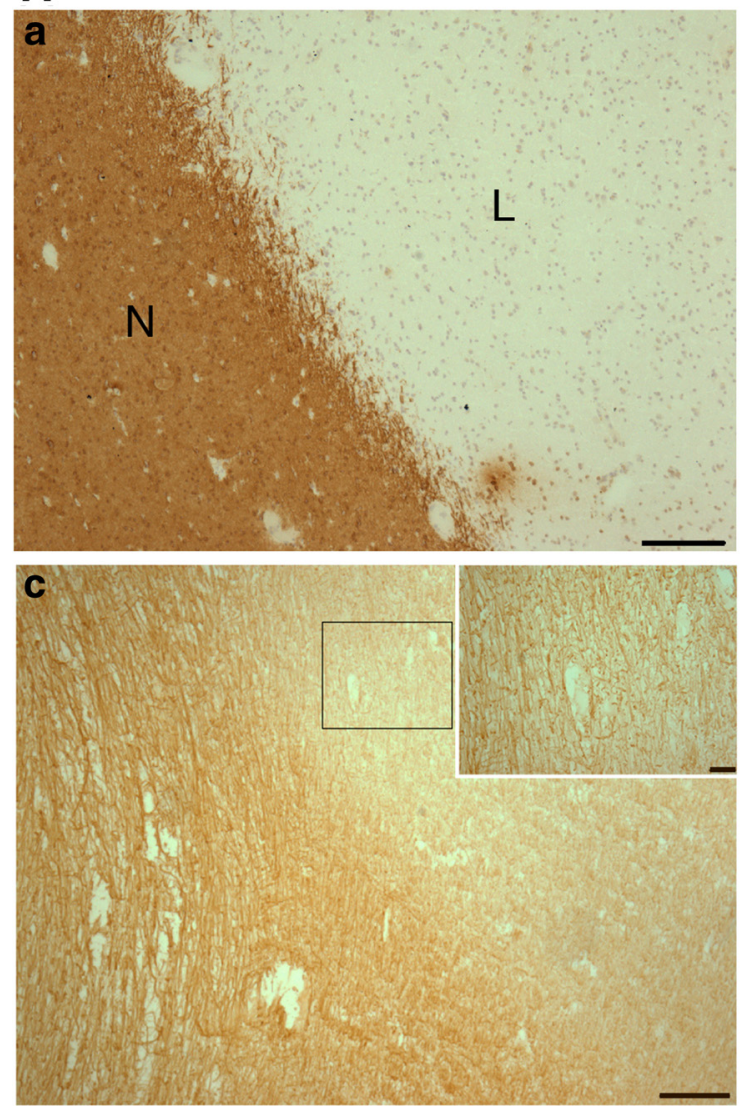

C

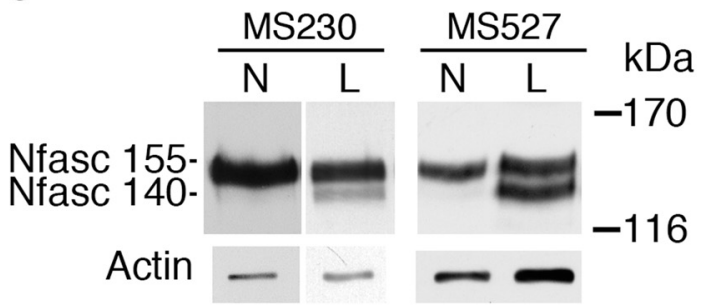

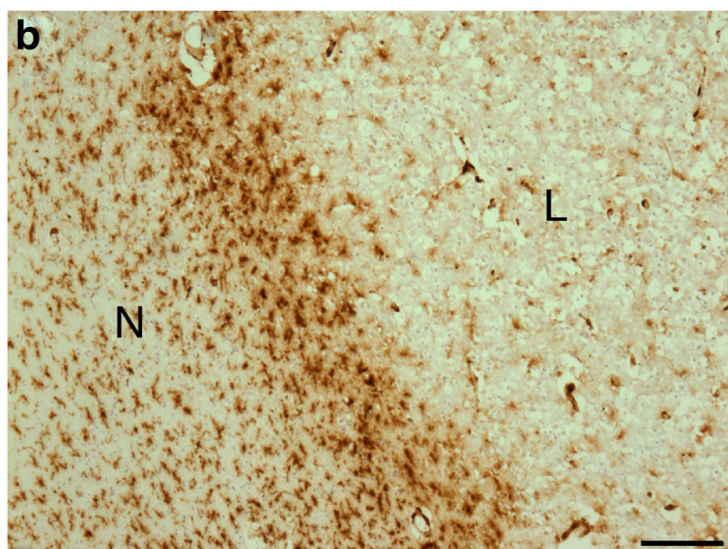

B

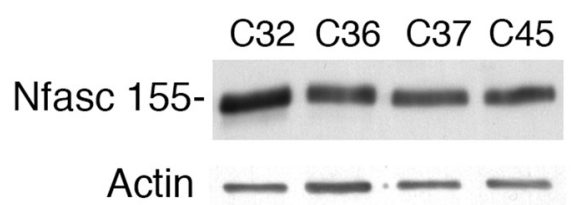

$$
\begin{array}{llll}
\text { MS } & \text { MS } & \text { MS } & \text { MS } \\
200 & 203 & 207 & 256
\end{array}
$$

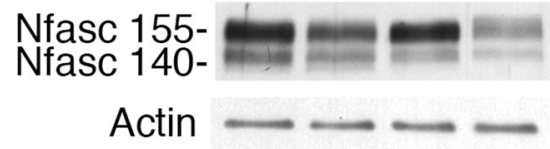

D

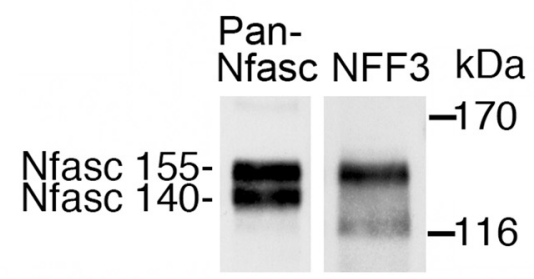

Figure 2. Nfasc140 is reexpressed in demyelinated lesions. Aa, Chronic active lesions ( $\mathrm{L}$ ) were defined by a lack of PLP staining compared with the normal-appearing white matter (N) and, as shown in $\boldsymbol{A} \boldsymbol{b}$, a rim of increased numbers of activated HLA Class II-positive microglia. $\boldsymbol{A c}$, Demyelinated axons, as visualized by neurofilament staining, persisted in the lesions, as also shown at higher magnification in the inset area delineated by the box. Scale bar: $100 \mu \mathrm{m}$; inset, $10 \mu \mathrm{m}$. B. Western blot using the NFC antibody (Pan-Nfasc) described in the legend to Figure 1 of lysates from the white matter of four different control subjects (C32, C36, C37, C45) and from chronic active lesions from four different MS patients (MS200, MS203, MS207, MS256) show the presence of Nfasc155, confirming the presence of oligodendrocytes but also revealing neuronal Nfasc140 expression in the lesions. Actin is the loading control. $\mathbf{C}$, Western blot comparison of normal-appearing white matter (N) and chronic active lesions (L) from two different subjects using the NFC antibody (Pan-Nfasc) described in the legend to Figure 1 show that Nfasc 140 is increased in the lesions. Actin is the loading control. $\boldsymbol{D}$, Western blots of chronic active lesion lysates from the MS2000 subject using the NFC antibody (Pan-Nfasc) described in the legend to Figure 1 and the antibody that recognizes the fourth FNIII domain specific for glial neurofascin (NFF3) confirm that the Nfasc 140 band is not a variant of Nfasc 155 . The lower band at $116 \mathrm{kDa}$ is probably neurofascin produced by ectodomain cleavage that lacks the $C$ terminus recognized by Pan-Nfasc as shown in Figure $1 C$.

\section{Nfasc140 is reexpressed in demyelinated MS lesions}

We have previously shown that demyelination in MS lesions leads to disruption of the nodal and paranodal domains (Howell et al., 2006). Because Nfasc140 is normally expressed strongly at the very earliest stages of myelination, we asked whether this neurofascin isoform might be reexpressed in chronic active lesions where demyelination was occurring. Lesions were identified as described in Materials and Methods (Fig. 2A). As expected, Nfasc140 is either not detectable or present at very low levels by Western blot in mature non-neurological control white matter (Fig. $2 B, C$ ). In contrast, chronic active lesions in four different MS patients reexpress a smaller form of neurofascin, with a mo- bility in SDS-PAGE identical to Nfasc140 (Fig. 2B). Although the levels of Nfasc186 were highly variable (data not shown), expression of the paranodal glial neurofascin, Nfasc155, persisted, indicating the presence of oligodendrocytes in the lesions (Fig. 2B). The variations in the relative amounts of Nfasc140 and Nfasc155 (the latter being a marker of oligodendrocytes) presumably reflect the fact that the MS patients were of varying age and disease duration and the fact that the samples were not from anatomically identical places. Comparison of normal-appearing white matter with adjacent chronic active lesions in two different subjects confirmed that this lower band was much more abundant in the lesions (Fig. 2C). Domain-specific antibodies supported the 
A

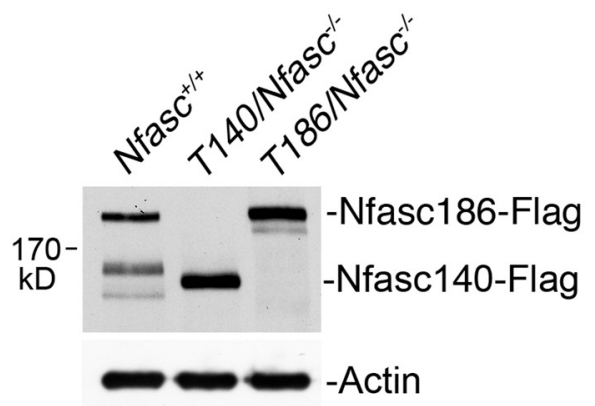

B
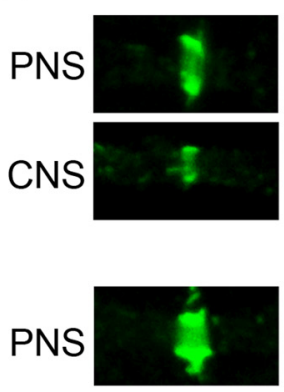

CNS

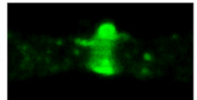

T140

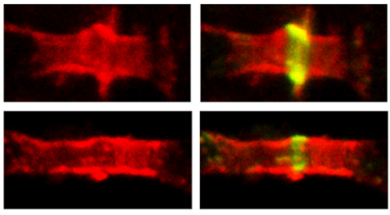

T186

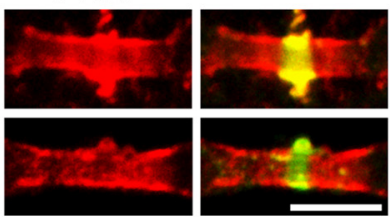

Nfasc140/Nfasc
C
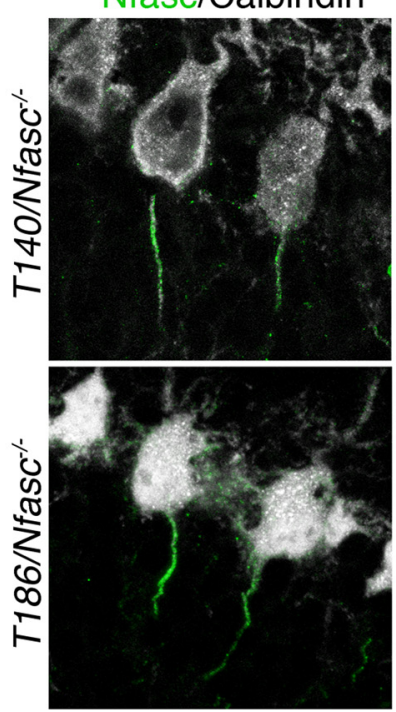

Flag/Calbindin

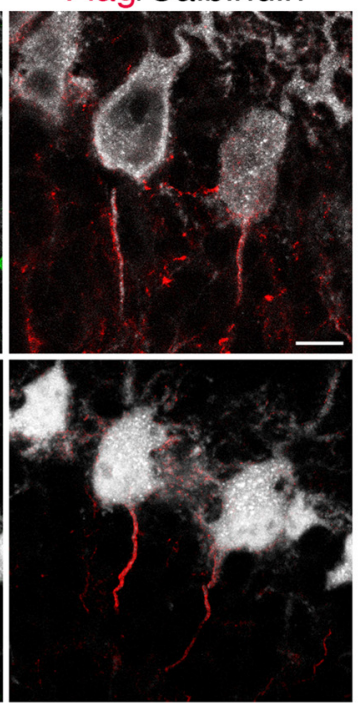

D

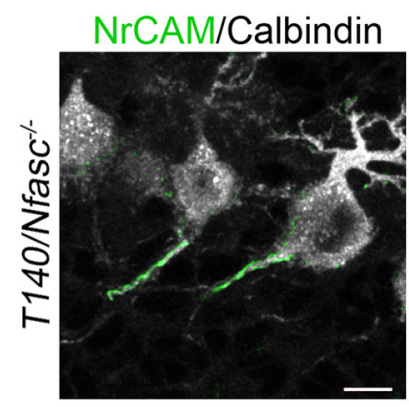

Figure 3. Transgenically expressed Nfasc 140 is targeted to nodes of Ranvier and the axon initial segment in neurons. $A$, Western blot analysis of spinal cord lysates from transgenic mice at P42 expressing either Nfasc 140 or Nfasc186 tagged at their C termini with FLAG on a neurofascin-null background and blotted with a pan anti-neurofascin antibody (NFC). Actin was the loading control. B, Immunostaining of teased quadriceps (PNS) or spinal cord ventral funiculus (CNS) fibers at P45 shows that both transgenic Nfasc 140 and Nfasc 186 are targeted to the node of Ranvier. Transgenic Nfasc140 is immunostained with an antibody against the FLAG epitope (green), and all neurofascins at the node (Nfasc140 and Nfasc186) and at the paranode (Nfasc155) are stained with an antibody that recognizes the C terminus of all full-length neurofascins (NFC) (red). Scale bar, $5 \mu \mathrm{m}$. C, Targeting of transgenic Nfasc 140 to the AIS of neurofascin-null cerebellar Purkinje cells at P8 is similar to that of Nfasc186. Immunostaining was for the C terminus of neurofascins (green), FLAG (red), and calbindin (white). D, Nfasc140 can recruit NrCAM to the AIS of cerebellar Purkinje cells of neurofascin-null mice at P8. Immunostaining was for the NrCAM (green) and calbindin (white). Scale bar, $10 \mu \mathrm{m}$.

view that this lower band was indeed Nfasc140 (Fig. 2D) and not a variant of Nfasc155 as has been detected in the brains of MS patients (Pomicter et al., 2013).

\section{Nfasc140 is targeted to the node of Ranvier and axon initial segment}

To study the function of Nfasc140, it was necessary to use a transgenic approach because this neurofascin isoform does not possess any unique domains not also found in Nfasc186 and Nfasc155 that would permit either the generation of isoform-specific antibodies, as is the case for Nfasc155 and Nfasc186, or an RNAi knockdown approach (Tait et al., 2000). To investigate the expression of the transgenically expressed protein, we attached a C-terminal FLAG-tag.

We generated transgenic lines expressing Nfasc140 with a C-terminal FLAG-tag, and one founder (T140) was selected in which FLAG-tagged Nfasc140 was robustly and ubiquitously expressed in neurons in both the CNS and PNS in similar fashion to the T186 transgenic line expressing the FLAG-tagged Nfasc186
(T186) we have described previously (Zonta et al., 2011). The level of expression of transgenically expressed Nfasc140 and Nfasc186 in the CNS was comparable, and the sizes of the proteins from Western blots were as predicted (Fig. $3 A$ ).

Because Nfasc 140 is a neuronal neurofascin isoform, we speculated that, like Nfasc186, it might also be targeted to the node of Ranvier (Tait et al., 2000). Immunofluorescence labeling showed that FLAG-tagged Nfasc140 was indeed concentrated at nodes both in the PNS and the CNS in comparable fashion with Nfasc186 in wild-type (WT) mice (Fig. 3B). We then asked whether Nfasc140 was, like Nfasc186, also targeted to AISs where Nfasc186 is known to play a crucial role in stabilizing the complex of proteins, including voltage-gated sodium channels in the adult cerebellum (Zonta et al., 2011). Nfasc140 was targeted to the AIS in $\mathrm{Nfasc}^{-/-}$mice (Fig. 3C). Further, because the absence of neuronal neurofascin during development causes the concomitant loss of the cell adhesion molecule NrCAM (Zonta et al., 2011), we asked whether Nfasc140 was able, like Nfasc186, to rescue 
A

NfasclC

NfasclCFlag

AnkBD

B

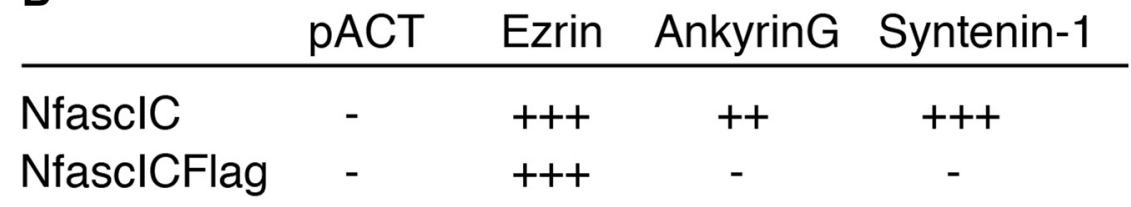

Figure 4. Introduction of the FLAG tag at the ( terminus of Nfasc186 or Nfasc140 prevents interaction with ankyrinG. $\boldsymbol{A}$, The 109 amino acid cytoplasmic tail common to Nfasc186, Nfasc140, and Nfasc155 (NfasclC) containing AnkBD (Zhang et al., 1998) was used as bait in a yeast two-hybrid interaction with empty vector (pAS2-1) or clones encoding regions of ezrin, ankyrinG, and syntenin-1 that had previously been identified as interactors (Davis and Bennett, 1994; Koroll et al., 2001; Gunn-Moore et al., 2006). $\boldsymbol{B}$, The degree of interaction was scored $(-,+,++,+++)$. NfasclCFlag was no longer capable of interacting with ankyring.

NrCAM expression at the AIS in neurofascin-null mice, which it did (Fig. 3D) (Zonta et al., 2011).

It has been proposed that the ankyrin binding site (AnkBD) in the C-terminal domain of the neurofascins assists in the recruitment of ankyrinG to the node of Ranvier (Davis and Bennett, 1994; Lambert et al., 1997). Therefore, we wished to test whether the AnkBD domain was still functional when a FLAG tag was present at the $\mathrm{C}$ terminus. We used the yeast two-hybrid technique to test the interaction of the intracellular domain common to Nfasc186, Nfasc140, and Nfasc155, with or without the presence of the FLAG epitope (Fig. 4A). The ankyrinG clone comprised amino acids 464759, previously isolated using NfascIC as bait (unpublished data), and the controls were clones encoding regions of ezrin and syntenin-1 that have previously been shown to be interactors with the neurofascin C terminus (Koroll et al., 2001; Gunn-Moore et al., 2006).

The prediction was that the FLAG-tag would not affect interaction with the ezrin-binding domain because this lies close to the transmembrane segment, although because syntenin-1 interacts with the PDZ-binding motif at the extreme $\mathrm{C}$ terminus of the neurofascins, interaction with syntenin-1 would be abolished (Koroll et al., 2001; Gunn-Moore et al., 2006). As predicted, interaction of the C-terminal domain of tagged neurofascin with ezrin was unaffected, whereas syntenin-1 no longer bound when the FLAG tag was at the C terminus (Fig. 4B). However, and perhaps surprisingly, the FLAG-tag also inhibited interaction of ankyrin with the AnkBD in this assay (Fig. 4B). Unfortunately, it was not possible to confirm this interaction by coimmunoprecipitation because the presence of the FLAG tag blocked the activity of the best antibody for the purpose, namely, the NFC antibody. Hence, this must remain a tentative conclusion. Nevertheless, it was interesting that the presence of the FLAG does not prevent targeting of both Nfasc140 and Nfasc186 to the AIS and nodes, which suggests that a direct interaction between neuronal neurofascins and ankyrinG may not be essential for node assembly.

\section{Nfasc140 rescues the nodal complex}

We then asked whether Nfasc140 FLAG-tagged at its C terminus could reconstitute the nodal complex in the axons of $\mathrm{Nfasc}^{-1-}$ mice in vivo in the absence of an intact axoglial adhesion complex. We have used this transgenic rescue approach previously to show that neuronal Nfasc186 and glial Nfasc155 have comple- geted appropriately (Fig. 5).

mentary functions in node assembly (Sherman et al., 2005; Zonta et al., 2008). We compared Nfasc140 with Nfasc186 because we knew the latter could rescue the nodal components on a neurofascinnull background (Sherman et al., 2005; Zonta et al., 2008). Expression of transgenic Nfasc140 on a neurofascin-null background was fully able to rescue the nodal complex in both the PNS and CNS (Fig. 5). Thus, sodium channels ankyrinG and $\beta I V$-spectrin were all targeted to CNS and PNS nodes by Nfasc140 similar to Nfasc186 (Fig. 5). Furthermore, the fact that we assessed targeting at P60 suggests that these nodal components are quite stable at the node in the presence of Nfasc140. NrCAM and gliomedin are PNS-specific nodal components, whereas brevican is CNS-specific, and all were tar-

The fact that the transgenic mice were null for Nfasc 155 meant that they could not have had intact paranodal axoglial junctions (Sherman et al., 2005; Zonta et al., 2008). This was reflected in the redistribution of Kv1.1 channels to a paranodal location from their normal juxtaparanodal domain (Fig. 5). However, this did not affect the ability of Nfasc140 (or Nfasc186) to recruit nodal proteins to the complex.

\section{Amelioration of nerve conduction velocity by $\mathrm{Nfasc} 140$ rescue of the PNS node}

Nerve conduction velocity measurements on quadriceps nerves showed that expression of Nfasc140 at PNS nodes restored nerve conduction velocities to the same degree as Nfasc186 (Fig. 6A) $\left(41.2 \pm 1.6 \mathrm{~m} / \mathrm{s}\right.$ for WT, $24.1 \pm 2.3$ for $\mathrm{T1}^{2} 40 / \mathrm{Nfasc}^{-1-}$, and $23.0 \pm 1.4$ for $\mathrm{T} 186 / \mathrm{Nfasc}^{-/-}$, values are mean \pm SEM; $n=6$ mice for each condition; $p<0.0001$, one-way ANOVA test followed by Tukey's Multiple Comparison Test) (Fig. 6A). Interestingly, it has been previously shown that, when axoglial junctions are disrupted by the loss of Caspr, conduction velocities in the PNS are 59\% of WT (Bhat et al., 2001). This agrees remarkably well with the values for nerves in which nodes were rescued by Nfasc140 (59\%) or Nfasc186 (56\%), where in both cases paranodal axoglial junctions were deranged due to the absence of Nfasc155 (Fig. 5) (Sherman et al., 2005). Although still not at WT values, these conduction velocities are a considerable improvement on the $81 \%$ reduction in the speed of conduction in the peripheral nerves of neurofascin-null mice, which die at P7 (Sherman et al., 2005). Consistent with their electrophysiological deficits, neurofascin-null mice rescued with Nfasc140 or Nfasc186 performed poorly on the rotarod motor behavioral test (Fig. 6B). Nevertheless, it was noteworthy that, even though the "rescued" mice had significant gait problems, they were otherwise healthy and survived up to 9 months of age after which they were killed.

\section{Discussion}

Our data provide further evidence for the essential role played by the Neurofascin gene in the assembly of the node of Ranvier, a key axonal domain in myelinated nerves. Hence, three isoforms of the same protein expressed in two distinct cell types cooperate to achieve the goal of clustering a macromolecular axonal complex, namely, voltage-gated sodium channels and its associated pro- 

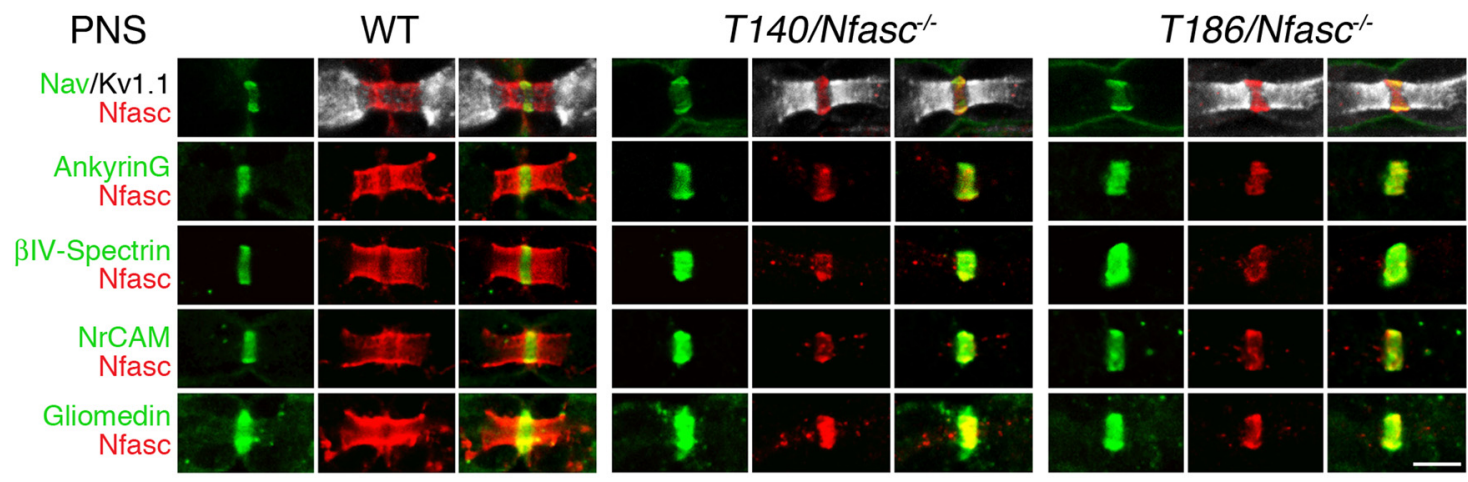

\section{CNS}
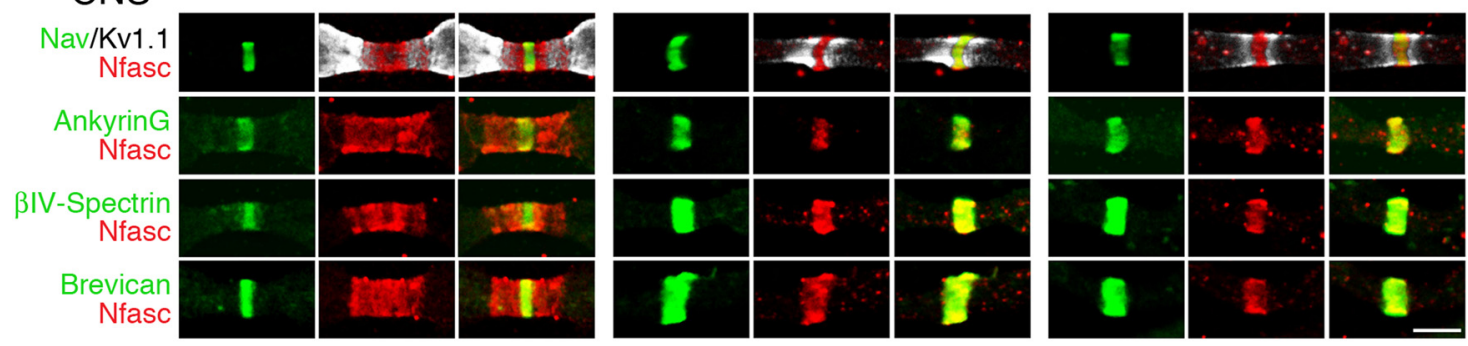

Figure 5. Nfasc 140 rescues the PNS and CNS nodal complexes as efficiently as Nfasc 186 in Nfasc ${ }^{-1}$ - mice. Immunostaining of teased quadriceps (PNS) and spinal cord ventral funiculus fibers (CNS) at P60 shows that both FLAG-tagged Nfasc140 and Nfasc186 can rescue the nodal components, voltage-gated sodium channels (Nav), ankyrinG, and $\beta$ IV-spectrin when expressed on a neurofascin-null background. Additionally, the PNS-specific nodal proteins NrCAM and gliomedin were also rescued, as was the CNS-specific nodal protein brevican. Rescue of the node was not accompanied by reformation of the axoglial junction; and, as a consequence, juxtaparanodal Kv1.1 channels were mislocalized compared with WT. Scale bars, $5 \mu \mathrm{m}$.

teins. This represents an unusual example of functionally cooperative, cell-typespecific alternative splicing of a single gene in cell biology. In the case of the neurofascins, this cooperativity results in the assembly of an essential structure in the development of the vertebrate nervous system.

Further, when considering the function of the rescued mice, we have previously found that neurofascin-null mice rescued with Nfasc186 survive into adulthood (Zonta et al., 2011). We also found that the same was true for mice rescued with the Nfasc140 transgene. This is quite surprising given the fact that both types of mice lack intact paranodal axoglial junctions. This may be due to the fact that the heterologous promoter used to drive expression of the Nfasc transgenes, namely, Thy1.2, remains active throughout adult life. In contrast, the endogenous neuronal Nfasc promoter is probably downregulated in adulthood due to the fact that Nfasc186, at least, is a very stable protein, which turns over very slowly at the node of Ranvier (Zonta et al., 2011).

The Neurofascin gene encodes six Ig repeats, five FNIII repeats, and a mucin domain (Davis et al., 1996) (Fig. 1). All three major neurofascin isoforms have the six Ig repeats; however, Nfasc186 has FNIII-1, 2, 4, and 5 repeats and the mucin domain, whereas Nfasc155 lacks the mucin domain and has the FNIII-1, 2, 3, and 4 repeats. Therefore, the mucin and FNIII- 5 domains are unique to neuronal Nfasc186, whereas the FNIII-3 domain is unique to
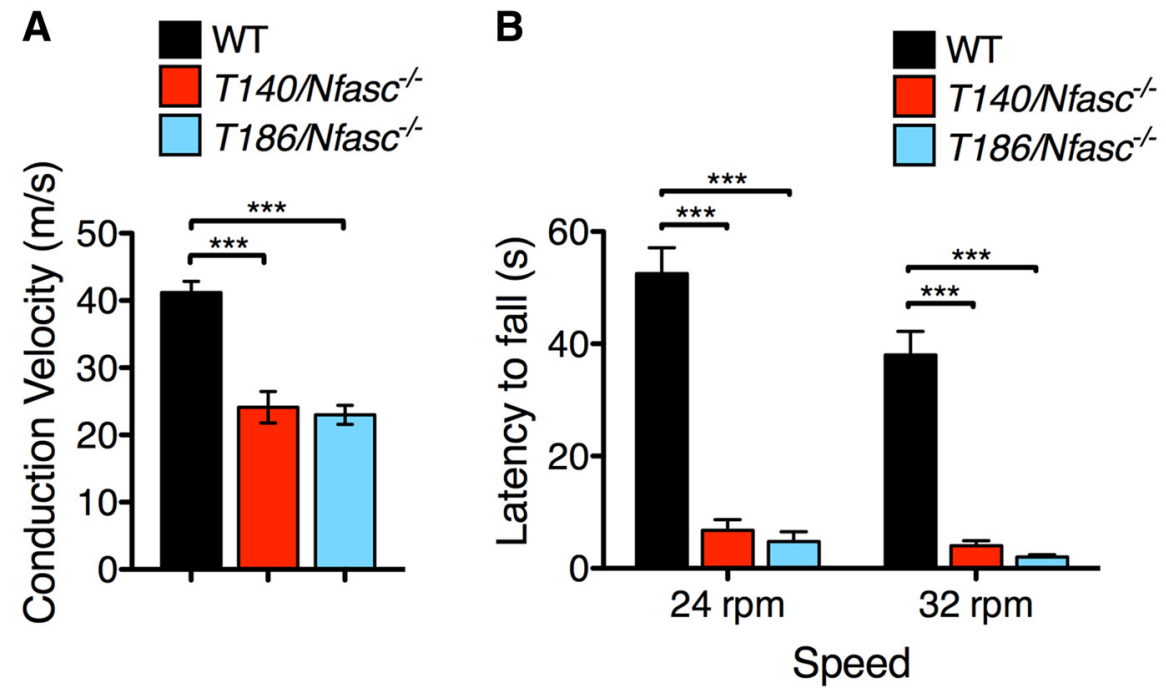

Figure 6. Nfasc140 restores limited nodal function in 2-month-old $\mathrm{Nfasc}^{-1-}$ mice. $A$, Conduction velocities were measured in quadriceps nerves from mice at P60. Although significantly less than WT mice, values from mice expressing Nfasc 140 were not significantly different from those expressing Nfasc 186. B, T140/Nfasc ${ }^{-1-}$ and $1186 / \mathrm{Nfasc}^{-1-}$ P60 animals were significantly worse than WT mice on the rotarod. And the deficit comparing the two different transgenic animals was not significantly different. Data are mean \pm SEM $; n=6$ mice for electrophysiology and $n=4$ for rotarod. ${ }^{* * *} p<0.0001$ (one-way ANOVA test followed by Tukey's Multiple Comparison Test).

glial Nfasc155. In contrast, Nfasc140 has the FNIII-1, 2, and 4 repeats found in Nfasc186 but lacks FMIII- 5 and the mucin domain. The fact that Nfasc 140 lacks these domains shows that they are neither required for targeting of $\mathrm{Nfasc} 140$ to the node nor are they necessary for sodium channel clustering. It remains to be determined what the function of these domains might be. Other work has shown that Ig5-6 are also dispensable for Nfasc186 targeting to the node (Thaxton et al., 2010), although the ectodomain of Nfasc186 is necessary for targeting to the PNS node 
(Dzhashiashvili et al., 2007). Further, although there is evidence that ankyrinG promotes sodium channel clustering at the PNS node (Dzhashiashvili et al., 2007), our data show that interaction of ankyrinG with either Nfasc186 or Nfasc140 is not necessary for either targeting or maintenance of sodium channels at the node in both the CNS and PNS.

Nfasc140 appears to have a similar structure and developmental pattern of expression to NF166, previously described in chick (Pruss et al., 2006). The early expression of Nfasc140 during development suggests that $\mathrm{Nfasc} 140$ complements the function of Nfasc155 and Nfasc186 in the initial stages of the assembly and stabilization of the nodal complex. Further, Nfasc140 is reexpressed in demyelinated regions of postmortem brain tissue from human subjects with MS. Our previous studies demonstrated that Nfasc186 expression was disrupted in MS white matter lesions and became diffusely distributed along demyelinated axons in a manner similar to sodium channels (Howell et al., 2006). The demonstration that Nfasc140, which is normally absent from adult axons, becomes reexpressed in demyelinated lesions suggests a return to a premyelinating state, perhaps in preparation for remyelination to occur. This is in keeping with the reduced expression of Nfasc155 in the same demyelinated lesions, indicating the presence of some oligodendrocytes or residual myelin.

In conclusion, this work expands the critical role of the Nfasc gene in the function of myelinated axons and reveals further redundancy in the mechanisms required for the formation of this crucial structure in the vertebrate nervous system.

\section{References}

Bekku Y, Oohashi T (2010) Neurocan contributes to the molecular heterogeneity of the perinodal ECM. Arch Histol Cytol 73:95-102. CrossRef Medline

Berghs S, Aggujaro D, Dirkx R Jr, Maksimova E, Stabach P, Hermel JM, Zhang JP, Philbrick W, Slepnev V, Ort T, Solimena M (2000) betaIV spectrin, a new spectrin localized at axon initial segments and nodes of ranvier in the central and peripheral nervous system. J Cell Biol 151:9851002. CrossRef Medline

Bhat MA, Rios JC, Lu Y, Garcia-Fresco GP, Ching W, St Martin M, Li J, Einheber S, Chesler M, Rosenbluth J, Salzer JL, Bellen HJ (2001) Axonglia interactions and the domain organization of myelinated axons requires neurexin IV/Caspr/Paranodin. Neuron 30:369-383. CrossRef Medline

Boyle ME, Berglund EO, Murai KK, Weber L, Peles E, Ranscht B (2001) Contactin orchestrates assembly of the septate-like junctions at the paranode in myelinated peripheral nerve. Neuron 30:385-397. CrossRef Medline

Charles P, Tait S, Faivre-Sarrailh C, Barbin G, Gunn-Moore F, DenisenkoNehrbass N, Guennoc AM, Girault JA, Brophy PJ, Lubetzki C (2002) Neurofascin is a glial receptor for the paranodin/Caspr-contactin axonal complex at the axoglial junction. Curr Biol 12:217-220. CrossRef Medline

Collinson JM, Marshall D, Gillespie CS, Brophy PJ (1998) Transient expression of neurofascin by oligodendrocytes at the onset of myelinogenesis: implications for mechanisms of axon-glial interaction. Glia 23:11-23. CrossRef Medline

Court FA, Sherman DL, Pratt T, Garry EM, Ribchester RR, Cottrell DF, Fleetwood-Walker SM, Brophy PJ (2004) Restricted growth of Schwann cells lacking Cajal bands slows conduction in myelinated nerves. Nature 431:191-195. CrossRef Medline

Davis JQ, Bennett V (1993) Ankyrin-binding activity of nervous system cell adhesion molecules expressed in adult brain. J Cell Sci Suppl 17:109-117. Medline

Davis JQ, Bennett V (1994) Ankyrin binding activity shared by the neurofascin/L1/NrCAM family of nervous system cell adhesion molecules. J Biol Chem 269:27163-27166. Medline

Davis JQ, McLaughlin T, Bennett V (1993) Ankyrin-binding proteins related to nervous system cell adhesion molecules: candidates to provide transmembrane and intercellular connections in adult brain. J Cell Biol 121:121-133. CrossRef Medline
Davis JQ, Lambert S, Bennett V (1996) Molecular composition of the node of Ranvier: identification of ankyrin-binding cell adhesion molecules neurofascin (mucin +/third FNIII domain-) and NrCAM at nodal axon segments. J Cell Biol 135:1355-1367. CrossRef Medline

Dzhashiashvili Y, Zhang Y, Galinska J, Lam I, Grumet M, Salzer JL (2007) Nodes of Ranvier and axon initial segments are ankyrin G-dependent domains that assemble by distinct mechanisms. J Cell Biol 177:857-870. CrossRef Medline

Einheber S, Zanazzi G, Ching W, Scherer S, Milner TA, Peles E, Salzer JL (1997) The axonal membrane protein Caspr, a homologue of neurexin IV, is a component of the septate-like paranodal junctions that assemble during myelination. J Cell Biol 139:1495-1506. CrossRef Medline

Eshed Y, Feinberg K, Poliak S, Sabanay H, Sarig-Nadir O, Spiegel I, Bermingham JR Jr, Peles E (2005) Gliomedin mediates Schwann cell-axon interaction and the molecular assembly of the nodes of Ranvier. Neuron 47: 215-229. CrossRef Medline

Feinberg K, Eshed-Eisenbach Y, Frechter S, Amor V, Salomon D, Sabanay H, Dupree JL, Grumet M, Brophy PJ, Shrager P, Peles E (2010) A glial signal consisting of gliomedin and $\mathrm{NrCAM}$ clusters axonal $\mathrm{Na}^{+}$channels during the formation of nodes of Ranvier. Neuron 65:490-502. CrossRef Medline

Gunn-Moore FJ, Hill M, Davey F, Herron LR, Tait S, Sherman D, Brophy PJ (2006) A functional FERM domain binding motif in neurofascin. Mol Cell Neurosci 33:441-446. CrossRef Medline

Howell OW, Palser A, Polito A, Melrose S, Zonta B, Scheiermann C, Vora AJ, Brophy PJ, Reynolds R (2006) Disruption of neurofascin localization reveals early changes preceding demyelination and remyelination in multiple sclerosis. Brain 129:3173-3185. CrossRef Medline

Jenkins SM, Bennett V (2002) Developing nodes of Ranvier are defined by ankyrin-G clustering and are independent of paranodal axoglial adhesion. Proc Natl Acad Sci U S A 99:2303-2308. CrossRef Medline

Kazarinova-Noyes K, Malhotra JD, McEwen DP, Mattei LN, Berglund EO, Ranscht B, Levinson SR, Schachner M, Shrager P, Isom LL, Xiao ZC (2001) Contactin associates with $\mathrm{Na}^{+}$channels and increases their functional expression. J Neurosci 21:7517-7525. Medline

Koroll M, Rathjen FG, Volkmer H (2001) The neural cell recognition molecule neurofascin interacts with syntenin-1 but not with syntenin-2, both of which reveal self-associating activity. J Biol Chem 276:10646-10654. CrossRef Medline

Lambert S, Davis JQ, Bennett V (1997) Morphogenesis of the node of Ranvier: co-clusters of ankyrin and ankyrin-binding integral proteins define early developmental intermediates. J Neurosci 17:7025-7036. Medline

Leone DP, Genoud S, Atanasoski S, Grausenburger R, Berger P, Metzger D, Macklin WB, Chambon P, Suter U (2003) Tamoxifen-inducible gliaspecific Cre mice for somatic mutagenesis in oligodendrocytes and Schwann cells. Mol Cell Neurosci 22:430-440. CrossRef Medline

Lüthi A, Van der Putten H, Botteri FM, Mansuy IM, Meins M, Frey U, Sansig G, Portet C, Schmutz M, Schröder M, Nitsch C, Laurent JP, Monard D (1997) Endogenous serine protease inhibitor modulates epileptic activity and hippocampal long-term potentiation. J Neurosci 17:4688-4699. Medline

Mahad DJ, Ziabreva I, Campbell G, Lax N, White K, Hanson PS, Lassmann H, Turnbull DM (2009) Mitochondrial changes within axons in multiple sclerosis. Brain 132:1161-1174. CrossRef Medline

Maier O, van der Heide T, Johnson R, de Vries H, Baron W, Hoekstra D (2006) The function of neurofascin 155 in oligodendrocytes is regulated by metalloprotease-mediated cleavage and ectodomain shedding. Exp Cell Res 312:500-511. CrossRef Medline

Menegoz M, Gaspar P, Le Bert M, Galvez T, Burgaya F, Palfrey C, Ezan P, Arnos F, Girault JA (1997) Paranodin, a glycoprotein of neuronal paranodal membranes. Neuron 19:319-331. CrossRef Medline

Pomicter AD, Deloyht JM, Hackett AR, Purdie N, Sato-Bigbee C, Henderson SC, Dupree JL (2013) Nfasc155H and MAG are specifically susceptible to detergent extraction in the absence of the myelin sphingolipid sulfatide. Neurochem Res 38:2490-2502. CrossRef Medline

Pruss T, Kranz EU, Niere M, Volkmer H (2006) A regulated switch of chick neurofascin isoforms modulates ligand recognition and neurite extension. Mol Cell Neurosci 31:354-365. CrossRef Medline

Reynolds R, Roncaroli F, Nicholas R, Radotra B, Gveric D, Howell O (2011) The neuropathological basis of clinical progression in multiple sclerosis. Acta Neuropathol 122:155-170. CrossRef Medline 
Salzer JL, Brophy PJ, Peles E (2008) Molecular domains of myelinated axons in the peripheral nervous system. Glia 56:1532-1540. CrossRef Medline

Sherman DL, Brophy PJ (2005) Mechanisms of axon ensheathment and myelin growth. Nat Rev Neurosci 6:683-690. CrossRef Medline

Sherman DL, Fabrizi C, Gillespie CS, Brophy PJ (2001) Specific disruption of a schwann cell dystrophin-related protein complex in a demyelinating neuropathy. Neuron 30:677-687. CrossRef

Sherman DL, Tait S, Melrose S, Johnson R, Zonta B, Court FA, Macklin WB, Meek S, Smith AJ, Cottrell DF, Brophy PJ (2005) Neurofascins are required to establish axonal domains for saltatory conduction. Neuron 48: 737-742. CrossRef Medline

Sherman DL, Krols M, Wu LM, Grove M, Nave KA, Gangloff YG, Brophy PJ (2012) Arrest of myelination and reduced axon growth when Schwann cells lack mTOR. J Neurosci 32:1817-1825. CrossRef Medline

Tait S, Gunn-Moore F, Collinson JM, Huang J, Lubetzki C, Pedraza L, Sherman DL, Colman DR, Brophy PJ (2000) An oligodendrocyte cell adhesion molecule at the site of assembly of the paranodal axo-glial junction. J Cell Biol 150:657-666. CrossRef Medline

Thaxton C, Pillai AM, Pribisko AL, Labasque M, Dupree JL, Faivre-Sarrailh C, Bhat MA (2010) In vivo deletion of immunoglobulin domains 5 and 6 in neurofascin (Nfasc) reveals domain-specific requirements in myelinated axons. J Neurosci 30:4868-4876. CrossRef Medline

Thaxton C, Pillai AM, Pribisko AL, Dupree JL, Bhat MA (2011) Nodes of Ranvier act as barriers to restrict invasion of flanking paranodal domains in myelinated axons. Neuron 69:244-257. CrossRef Medline

Yang Y, Lacas-Gervais S, Morest DK, Solimena M, Rasband MN (2004) BetaIV spectrins are essential for membrane stability and the molecular organization of nodes of Ranvier. J Neurosci 24:9230-9240. Medline

Zhang X, Davis JQ, Carpenter S, Bennett V (1998) Structural requirements for association of neurofascin with ankyrin. J Biol Chem 273:3078530794. CrossRef Medline

Zonta B, Tait S, Melrose S, Anderson H, Harroch S, Higginson J, Sherman DL, Brophy PJ (2008) Glial and neuronal isoforms of Neurofascin have distinct roles in the assembly of nodes of Ranvier in the central nervous system. J Cell Biol 181:1169-1177. CrossRef Medline

Zonta B, Desmazieres A, Rinaldi A, Tait S, Sherman DL, Nolan MF, Brophy PJ (2011) A critical role for Neurofascin in regulating action potential initiation through maintenance of the axon initial segment. Neuron 69:945956. CrossRef Medline 